ARTICLE

\title{
Adolescent antiretroviral management: Understanding the complexity of non-adherence
}

\author{
K Naidoo, ${ }^{1,2}$ MB ChB; A Munsami, ${ }^{1}$ MSSC; M Archary, ${ }^{3} \mathrm{MB}$ ChB \\ ${ }^{1}$ Centre for the AIDS Programme of Research in South Africa (CAPRISA), University of KwaZulu-Natal, Durban, South Africa \\ ${ }^{2}$ MRC-CAPRISA HIV-TB Pathogenesis and Treatment Research Unit, Doris Duke Medical Research Institute, University of KwaZulu-Natal, \\ Durban, South Africa \\ ${ }^{3}$ Paediatric Infectious Disease Unit, King Edward VIII Hospital, University of KwaZulu-Natal, Durban, South Africa
}

Corresponding author: K Naidoo (kogie.naidoo@caprisa.org)

This case-based discussion highlights challenges in adolescent antiretroviral management, focusing on non-disclosure of status and the subsequent impact of suboptimal treatment adherence. Despite the scale-up of antiretroviral therapy (ART) and recommendations made by the World Health Organization (WHO) for ART for all human immunodeficiency virus (HIV)-infected paediatric patients, ART coverage in adolescents lags behind that in adults. Challenges of sustaining lifelong ART in children and adolescents require consideration of specific behavioural, physiological and psychosocial complexities associated with this special group. To preserve future drug options and sustain lifelong access to therapy, addressing non-adherence to treatment is critical to minimising acquisition of ART drug resistance and treatment failure. We review the psychosocial and developmental components that influence the course of the disease in adolescents and consider the complexities arising from perinatal exposure to ART and the growing risk of transmitted ART drug resistance in high-burden resourcelimited settings.

S Afr Med J 2015;105(11):953. DOI:10.7196/SAMJ.2015.v105i11.10150

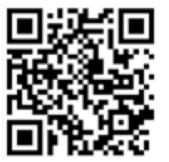

\section{Case report}

A 13-year-old male adolescent with severe respiratory distress presented to a medical outpatient department. He was admitted to the adult ward with suspected Pneumocyctis jirovecii pneumonia. He was known to be HIV-infected and on antiretroviral therapy (ART) for approximately 10 years, with a CD 4 count of 10 cells $/ \mu \mathrm{L}(0.1 \%)$ and a viral load of 1.5 million copies/mL (Fig. 1).

On further enquiry, it was noted that his mother had died from an HIV-related illness when he was 3 years old; he was subsequently adopted by his maternal aunt. History of nevirapine exposure for prevention of mother-to-child transmission (PMTCT) was not known. During a routine clinic visit, at age 3, he was diagnosed as HIV-positive and was commenced on ART (stavudine, lamivudine and efavirenz).

The patient's ART regimen was changed at his local clinic to second-line therapy (zidovudine, didanosine, lopinavir) when he was 5 years old. The reason for the change was not documented; however, during that time his aunt commenced shift work at night, and a neighbour was given the responsibility of administering medication to the patient for almost a year. After adherence counselling, his aunt resumed normal working hours and took responsibility for administering ART, after which the patient maintained HIV virological suppression to undetectable levels with reasonable CD4 count recovery, until 2 years ago.

As is common in our setting, the carer had not yet disclosed the HIV status to the patient. Here, the patient believed that his daily medication comprised antituberculosis drugs to prevent him from becoming 'sick'. A school friend pointed out that the medication was ART. His aunt had noticed initial subtle behavioural change, which progressed to overt aggression, violent tendencies towards his co-learners at school and substance abuse. He stopped taking ART and left his aunt's home.
During the course of the latest admission for P. jirovecii pneumonia, the patient and his aunt underwent extensive counselling and the former agreed to recommence ART (zidovudine, lamivudine, lopinavir/ritonavir), under supervision of his aunt. Six weeks later his viral load was 700000 copies $/ \mathrm{mL}$ and an HIV drug-resistance genotype test showed mutations to protease inhibitors (M46I, I54V, $V 82 A$ ), nucleoside reverse-transcriptase inhibitors (NRTIs) ( $M 41 L$, D67N, K70T, T215Y, K219Q) and non-nucleoside reverse-transcriptase inhibitors (NNRTIs) (K103N). During the course of infancy, childhood and adolescence, he had accumulated extensive mutations to all available classes of antiretroviral medications, limiting current and future treatment options. Based on the patient's resistance testing, he was commenced on tenofovir/emtricitabine (Truvada), daurinavir/ ritonavir and raltegravir. He currently remains virologically suppressed.

We present a case that highlights the complexity of managing long-term adolescent survivors of HIV acquired through vertical transmission. We describe the impact of non-disclosure of HIV status on medication adherence, and the impact of non-adherence on acquisition of ART drug resistance.

Urgent unmet needs in adolescent HIV care include the challenge of lifelong ART, limited safe, effective and robust drug options, and lack of co-formulated antiretroviral drugs appropriate for oncedaily dosing. ${ }^{[1]}$ While the development of a single-pill treatment regimen has resulted in high ART adherence and acceptability, with substantial improvements in quality of care in HIV-infected adults, this option is not yet available to children and adolescents. ${ }^{[2]}$

\section{What is the burden of disease in adolescents and children?}

Globally, adolescence is defined as the period of development between the ages of 10 and 19 years, represents the fastest growing age group of HIV-infected people, and accounts for $5.9 \%$ of the burden of HIV. ${ }^{[1]}$ Approximately 2500 of the 6300 new HIV infections that 


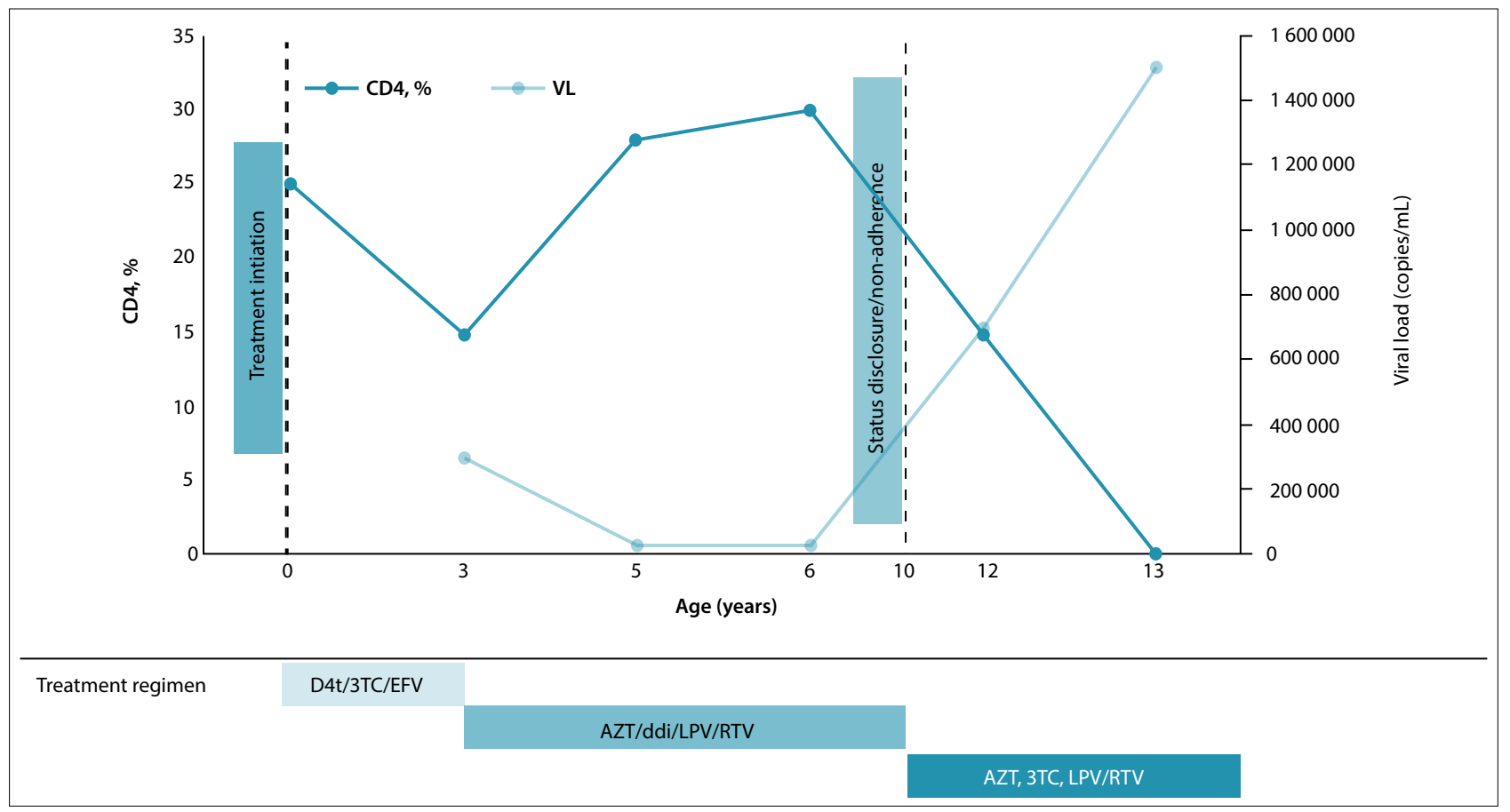

Fig. 1. CD4 count and viral load responses to ART during 13 years of follow-up of a child with vertically acquired $H I V(V L=v i r a l ~ l o a d ; D 4 t=$ stavudine; $3 T C=$ lamivudine; $E F V=$ efavirenz; $A Z T=$ zidovudine; ddi = didanosine; $L P V=$ lopinavir $; R T=$ ritonavir).

occur daily worldwide, are in adolescents and youth $15-24$ years of age, while a third of these are in children $<15$ years owing largely to vertical transmission. ${ }^{[1]}$ Temporal trends analysis of adolescent HIV prevalence conducted between 2006 and 2012 in South Africa (SA), while showing decreases from $22.4 \%$ to $19.3 \%$ (15 - 24 age group), $13.7 \%$ to $14.4 \%$ (15 - 19 age group) and $28.0 \%$ to $24.2 \%$ (20 - 24 age group), ${ }^{[3]}$ still remains alarmingly high. The expanding disease burden among adolescents underscores the need for a greater understanding and suitable adaptation of HIV care and treatment services to adequately meet the needs of this patient population.

Notwithstanding unprecedented investments in ART scale-up and delivery in the past decade, published literature shows that adolescents are less likely to access HIV care and remain in care or achieve virological suppression. ${ }^{[4]}$ Despite guidance in the management of adolescent ART issued by the WHO in 2013 there have, however, been serious impediments to reducing delays in the initiation of appropriate ART, in supporting adherence to treatment and in retention of adolescents in care. ${ }^{[1]}$ Currently, approximately $90 \%$ of the estimated 3 million children living with HIV globally ${ }^{[1]}$ reside in sub-Saharan Africa ${ }^{[5]}-$ only $27 \%$ of those who require ART receive it. ${ }^{[1]}$ Furthermore, in SA only $45 \%$ of the 369000 HIV-infected children aged 10 - 14 years, and $14.3 \%$ of the 720000 aged 15 - 24 years, currently receive ART. ${ }^{[6]}$ This may be due to HIV-exposed children often not receiving conclusive HIV test results, poor linkage to appropriate paediatric care, and lack of facilities that specifically cater for children. ${ }^{[1]}$

As in adults, the benefits of ART in HIV-positive children and adolescents include a decreased risk of death, improved growth, better immune function, and a marked reduction in infectious complications. ${ }^{[7]}$ The benefits and risks need to be considered in the context of safe and sustainable therapeutic options for lifelong ART. In 2013, WHO HIV guidelines recommended that adult ART regimens and dosing schedules be applied to adolescents $>35 \mathrm{~kg}$. These recommendations did not take into consideration the pubertal changes and growth delays that affect ART metabolism, a lack of clarity guiding the timing of transition from weight-based to age- based dosing, and a lack of efficacy and safety data on co-formulated agents. ${ }^{[8]}$ HIV resistance in children occurs as transmitted drug resistance (TDR), which is either vertical (from mother to child) or horizontal (through a sexual partner), or has been acquired, and results from poor ART adherence.

The US Reaching for Excellence in Adolescent Care and Health (REACH) study evaluating disease progression, which was conducted in HIV-positive adolescents infected through sexual behaviour or injection drug use, found that only $41 \%$ of adolescents aged 12 19 years reported $>95 \%$ adherence to ART. ${ }^{[9]}$ Factors associated with poor adherence included depression, pill burden, advanced HIV status, alcohol use, and dropping out of school. ${ }^{[10]}$ In addition, Murphy et al. ${ }^{[9]}$ reported that only $28.3 \%$ of adolescents reported $>95 \%$ adherence in the previous month. Barriers to adherence included ART toxicity and complications with regard to integrating pill-taking with day-to-day routines. ${ }^{[7,9]}$ In another US study, the Pediatric AIDS Clinical Trial Group (PACTG) 381, of 120 adolescents (aged $11-22$ years) infected via high-risk behaviour and receiving triple ART therapy, only 44 (37\%) stayed on study treatment for the 3 years of observation. Twenty-nine (24\%) reached and maintained viral suppression, and poor adherence was the main predictor of virological failure. ${ }^{[11]}$

With increases in the number of adolescents on ART, sustaining optimal ART adherence has emerged as a major challenge to maintaining ongoing AIDS-free survival and prevention of sexual transmission for this group. Antiretroviral regimens are often complex, require good adherence for efficacy, and may lead to the development of viral resistance due to treatment non-adherence (defined as $\leq 95 \%$ of medication taken, or $>1$ missed dose per week) or suboptimal levels of antiretroviral agents.

Furthermore, in many HIV-endemic resource-limited settings, the future impact of perinatal HIV transmission strategies, including nevirapine or zidovudine mono- or dual therapy on acquisition of resistance to non-nucleoside-based first-line ART regimens, has not been fully quantified. Our case highlights themes that have already emerged from the literature in developed countries. A meta-analysis 
on ART adherence among children, adolescents and young adults living with HIV found variable and suboptimal adherence rates. This was especially the case among adolescents, where adherence was highly dependent on complex relationships between individuals, their families, society and other treatment regimen-related factors. ${ }^{[10]}$

A recently published systematic review of 15 studies assessing correlates of ART adherence among adolescents in low- and middle-income countries, highlights several factors associated with challenges in ART adherence and potential areas for intervention. Emerging themes for suboptimal adherence across all studies include gender and knowledge of serostatus, influence of family structure, impact of onerous ART regimens - including route of administration - and attitudes about medication, healthcare and environmental factors, such as rural versus urban and missed appointments. ${ }^{[12]}$ Other studies cite the following psychosocial risk factors for poor ART adherence: being orphaned, mental health problems, changes of guardianship, and absence of parental and social support. ${ }^{[5]}$

As seen with the case presented, major hindrances to adolescent adherence are the lack of autonomy and reliance on adult caregivers to access care, complying with clinic visits, collecting medication and adhering to the dosage schedule. ${ }^{[1]}$ These are further confounded by issues surrounding status disclosure, autonomy and maturity. Decisions around HIV status disclosure differ in those with horizontally acquired infection compared with vertical infection. However, evidence suggests that non-disclosure is associated with various negative outcomes. ${ }^{[5]}$ Often, status disclosure is deferred until children approach cognitive maturity and puberty, but the decision to disclose is dependent on the family and caregivers, especially as adolescent status disclosure simultaneously discloses the status of the biological mother ${ }^{[5,11]}$ Non-adherence to ART is further complicated by behavioural experimentation typical of this phase of development. The ability of HIV-infected adolescents to successfully transition from reliance on adults towards autonomy in taking responsibility for their illness, managing adherence to drugs and visiting clinics is further complicated during this stage of development by the display of behavioural patterns that deviate from expected or prescribed patterns, ${ }^{[5]}$ making close observation and interventions for non-adherence necessary. ${ }^{[1]}$

The recently revised 2015 SA Paediatric ART treatment guidelines advocate an abacavir-based regimen for first-line ART in children $<15$ years or $<40 \mathrm{~kg}$, which may then be changed to tenofovir disoproxil fumarate at 15 years of age and weight $\geq 40 \mathrm{~kg}$ in those with an undetectable viral load. The use of abacavir-based regimens in paediatric populations is thought to aid in slowing down acquired drug resistance, especially in children who require extended periods of ART owing to the high genetic barrier to resistance ${ }^{[11]}$ and favourable cross-resistance pattern. Conversely, a multicohort analysis of early virological response in 9453 SA children $<16$ years of age showed lower virological suppression rates at 6 months in those receiving an abacavir-based lopinavir/ritonavircontaining regimen compared with those receiving a stavudine-based lopinavir/ritonavir-containing regimen $(54 \%$ v. $70 \%, p<0.0001){ }^{[13]}$ Furthermore, alternative agents such as tenofovir disoproxil fumarate, while recommended for use in children $>2$ years of age by the US Food and Drug Administration, are currently registered in SA for use in patients $>12$ years of age owing to safety concerns and lack of available paediatric formulations. ${ }^{[14]}$ The WHO has, however, highlighted the risk of tenofovir-related renal and bone toxic effects, and recommends specific monitoring of children and prepubertal adolescents receiving tenofovir. ${ }^{[5]}$ Tenofovir alafenamide, a novel tenofovir prodrug currently being evaluated in Phase $2 \mathrm{~b}$ and 3 trials in adolescents, achieves a 90\% reduction in plasma tenofovir concentrations. This drug has also shown improved renal and bone safety profiles, while maintaining equivalent virologial suppression rates in adults compared with tenofovir disoproxil fumarate-containing regimens. ${ }^{[2]}$
Recent ART scale-up in SA has allowed more people to access care, but may inevitably engender HIV drug resistance, thereby limiting the benefits of treatment. Increases in paediatric HIV drug resistance in resource-limited settings have been driven by limited access to routine viral load monitoring, limited availability of paediatric drugs for second-line therapy and complexities related to PMTCT, paediatric care and ART adherence, as seen in the presented case. ${ }^{[1]}$ A recently published review of drug-resistant mutation prevalence rates after first-line ART failure among children in resource-limited settings, showed mutation rates of $80 \%$ for NRTIs, $88 \%$ for NNRTIs and $54 \%$ for boosted protease inhibitors. ${ }^{[15]}$

A survey conducted in 18 African countries reported an increase in TDR, primarily driven by NNRTI resistance, with moderate levels of TDR (5 - 15\%) being documented in KwaZulu-Natal. ${ }^{[15]}$ This is of concern, as this drug class forms the backbone of first-line ART regimens and prophylaxis for PMTCT. ${ }^{[15]}$

\section{What are the research gaps in understanding ART resistance in children?}

HIV-infected children and adolescents remain at high risk of disease progression and death. Insufficient attention has been directed towards the creation of specialised centres offering HIV testing and care. Evaluating the impact of services that prioritise the needs of children and adolescents in care on long-term ART adherence and retention is warranted. SA has recently celebrated tremendous success in improving PMTCT, offering all HIV-infected pregnant women immediate ART access. This implies that most children who were exposed to HIV during the perinatal period have also been exposed to maternal ART. The magnitude of HIV prevalence in the sexually active age group and the growing risk of transmitted ART resistance emphasise the need for research into the use of pre-ART resistance testing in children and adolescents initiating or changing ART. Observation of acquisition rates of ART resistance is especially important to inform future drug sequencing, especially in high-burden settings offering a programmatic approach.

\section{References}

1. World Health Organization (WHO). HIV and Adolescents: Guidance for HIV Testing and Counselling and Care for Adolescents Living with HIV: Recommendations. Geneva: WHO, 2013

2. Jarcho JA, Gandhi M, Gandhi RT. Single-pill combination regimens for treatment of HIV-1 infection N Engl J Med 2014;371(3):248-259. [http://dx.doi.org/10.1056/NEJMct1215532]

3. National Department of Health (NDOH). The National Antenatal Sentinel HIV and Syphilis Prevalence Survey, South Africa. 2005-2012. Pretoria: NDOH, 2013.

4. Lindsey JC, Bosch RJ, Rudy BJ, Flynn PM. Early patterns of adherence in adolescents initiating highly active antiretrovial therapy predict long-term adherence, virologic, and immunologic control. AIDS Patient Care STDs 2009;23(10):799. [http://dx.doi.org/10.1089/apc.2009.0081]

5. Lowenthal ED, Bakeera-Kitaka S, Marukutira T, Chapman J, Goldrath K, Ferrand RA. Perinatally acquired HIV infection in adolescents from sub-Saharan Africa: A review of emerging challenges. ancet Infect Dis 2014;14(7):627-639. [http://dx.doi.org/10.1016/S1473-3099(13)70363-3]

6. Shisana OR, Simbayi LC, Zuma K, et al. South African National HIV Prevalence, Incidence and Shisana OR, Simbayi LC, Zuma K, et al. South Af
Behaviour Survey, 2012. Pretoria: HSRC Press, 2014.

Behaviour Survey, 2012. Pretoria: HSRC Press, 2014.
Nachman SA, Stanley K, Yogev R, et al. Nucleoside analogs plus ritonavir in stable antiretroviral Nachman SA, Stanley K, Yogev R, et al. Nucleoside analogs plus ritonavir in stable antiretrovira
therapy - experienced HIV-infected children: A randomized controlled trial. JAMA 2000;283(4):492498. [http://dx.doi.org/10.1001/jama.283.4.492]

8. Nachman S, Zheng N, Acosta EP, et al. Pharmacokinetics, safety, and 48-week efficacy of oral raltegravi in HIV-1-infected children aged 2 through 18 years. Clin Infect Dis 2014;58(3):413-422. [http://dx.doi org/10.1093/cid/cit696]

9. Murphy DA, Wilson C, Durako S, Muenz L, Belzer M. Antiretroviral medication adherence among the REACH HIV-infected adolescent cohort in the USA. AIDS Care 2001;13(1):27-40. [http://dx.doi. org/10.1080/09540120020018161]

10. Kahana SY, Rohan J, Allison S, Frazier TW, Drotar D. A meta-analysis of adherence to antiretrovira therapy and virologic responses in HIV-infected children, adolescents, and young adults. AIDS Behav 2013;17(1):41-60. [http://dx.doi.org/10.1007/s10461-012-0159-4]

11. Levin L Abacavir in children: Abacavir S Afr J HIV Med 2002,3(3):19-22.

12. Hudelso $C$ Cher $L$ Factors asociated with adherence to antiret living with HIV/AIDS in low-and middle-income countries: A systematic review. AIDS Care living with HIV/AIDS in low-and middle-income countries: A syste
2015;27(7):805-816. [http://dx.doi.org/10.1080/09540121.2015.1011073]

13. Technau K-G, Schomaker M, Kuhn L, et al. Virologic response in children treated with abacavir13. Technau K-G, Schomaker M, Kuhn L, et al. Virologic response in children treated with abacavir-
compared with stavudine-based antiretroviral treatment: A South African multi-cohort analysis. Pediatr Infect Dis J 2014;33(6):617-622. [http://dx.doi.org/10.1097/INF.0000000000000222]

14. Food and Drug Administration. Antiretroviral drugs used in the treatment of HIV infection. http:// www.fda.gov/ForPatients/Illness/HIVAIDS/Treatment (accessed 2 October 2015).

15. Hamers RL, Sigaloff KC, Wensing AM, et al. Patterns of HIV-1 drug resistance after first-line antiretroviral therapy (ART) failure in 6 sub-Saharan African countries: Implications for second-line ART strategies. Clin Infect Dis 2012;54(11):1660-1669. [http://dx.doi.org/10.1093/cid/cis254] 